\title{
Ceftaroline in the treatment of methicillin-resistant and daptomycin-non-susceptible Staphylococcus aureus bacteremia and infective endocarditis in end-stage renal disease
}

\author{
Kristen Fuhrmann Pharm D, Richard Winn MD, Paula McKenzie MD, Mamoun Bashir MD
}

\begin{abstract}
As bacteria evolve and become resistant to preferred antibiotics, we often have to resort to alternative, second-line agents for treatment. This case highlights a growing trend being observed among methicillin-resistant Staphylococcus aureus (MRSA) isolates, in which the minimum inhibitory concentration breakpoints start to increase and traditional therapy fails. When clinicians are faced with a difficult to treat infection and constrained by declining renal function, drug allergies, or clinical scenario, it may be prudent to use therapy that is supported by only in vitro data, animal models, and strong case reports. Combining knowledge of the drug's kinetic properties, mechanism of action, safety, and adverse effect profile, and literature to support its use helped guide our decision in treating a patient with persistent MRSA bacteremia and infective endocarditis.
\end{abstract}

Key words: Methicillin-resistant Staphylococcus aureus, vancomycin, daptomycin, ceftaroline

\section{Case Presenttion}

A 34-year-old man presented to the emergency center with nausea, vomiting, and shortness of breath for two days. Past medical history included uncontrolled diabetes mellitus, end-stage renal disease on intermittent hemodialysis, failed kidney transplantation, and hypertension. The patient was previously admitted to our facility one month prior due to line sepsis secondary to methicillin-resistant Staphylococcus aureus (MRSA) bacteremia for which vancomycin

Corresponding author: Kristen Fuhrmann Pharm D Contact Information: kristen.fuhrmann@umchealthsystem.com DOI: 10.12746/swrccc2013.0104.048 (minimum inhibitory concentration $[\mathrm{MIC}]<1 \mathrm{mcg} / \mathrm{mL}$ ) was initiated and continued for two weeks on an outpatient basis. On admission the patient had an elevated white count with left shift, fever, and tachycardia. The patient was admitted to the intensive care unit, cultures were drawn prior to initiating antimicrobial therapy, and an empiric regimen of cefepime, piperacillin-tazobactam, and vancomycin was started. Due to his recent bacteremia, a transesophageal echocardiogram (TEE) was ordered to determine presence of cardiac vegetations. Initial blood cultures (2/2) grew MRSA (vancomycin MIC $<1 \mathrm{mcg} / \mathrm{mL}$, daptomycin MIC $<2 \mathrm{mcg} / \mathrm{mL}$ ) by the BD Phoenix automated system for antimicrobial susceptibility testing, and TEE revealed a 0.5 centimeter vegetation on the mitral valve. The infectious disease team was consulted at this time to manage MRSA endocarditis and persistent or relapsed bacteremia. Upon review of the vancomycin 
dosing and monitoring history, the infectious disease and nephrology team concluded that previous vancomycin dosing was inadequate and decided to continue with an optimized dosing regimen of vancomycin tailored to the patient's hemodialysis schedule and rifampin $300 \mathrm{mg}$ by mouth twice daily. Although there is no strong evidence to support the use of rifampin for native valve endocarditis, the decision was made to use it as adjunctive therapy for staphylococcal infection of the blood. Following a subsequent set of positive blood cultures (2/2) for MRSA and two more growing Gram positive cocci in clusters, the infectious disease team decided to discontinue vancomycin due to therapeutic non-response, based on persistent bacteremia and continued clinical deterioration even with therapeutic serum levels, and initiate daptomycin $6 \mathrm{mg} / \mathrm{kg}$ IV q48h plus rifampin. At this time susceptibilities for the repeat blood isolates were pending. Once available, susceptibilities were reviewed and showed the MIC for vancomycin had increased from 1 to $2 \mathrm{mcg} / \mathrm{mL}$ and daptomycin susceptibilities were reported as "N/R," which is interpreted as "non-susceptible", with MICs in the intermediate to possibly resistant range. Daptomycin was discontinued and following multiple case reports of its success as salvage therapy in MRSA bacteremia and endocarditis, the decision was made to initiate ceftaroline.

There are multiple case reports of similar scenarios, in which elevated and increasing vancomycin MICs (>2 mcg/mL) correspond with "N/R" or "nonsusceptible" daptomycin MICs for MRSA. These case reports highlight successful sterilization of blood within $<24$ hours of ceftaroline therapy, with suggested doses for endocarditis of 600 mg IV every 8 hours. However, there is limited dosing data for endocarditis in end-stage renal disease (ESRD) requiring hemodialysis. Available ESRD dosing exists for less invasive, FDA-approved indications only, such as acute bacterial skin and skin structure infections and communityacquired bacterial pneumonia (CABP). ${ }^{1,2,5}$ In a similar case reported by Zainah et al., a middle-aged man with persistent MRSA bacteremia and ESRD requiring hemodialysis was safely treated with ceftaroline 400 mg IV every 12 hours. Similarly, in vitro suscepti- bilities of MRSA isolates from the patient's blood culture showed the trend of increasing vancomycin MICs and corresponding increase in daptomycin MICs. TEE and transthoracic echo (TTE) did not show evidence of vegetation, indicating that the infection was less invasive than in our particular case. ${ }^{5}$ Based on safe and similar use in other patient cases, multiple case reports of its use as salvage therapy, and the cephalosporin's established wide safety profile, we started ceftaroline 600 mg IV every 12h, a slightly reduced frequency from the suggested every eight hour frequency in patients with adequate renal function. After two doses of ceftaroline $600 \mathrm{mg}$ plus continued rifampin, three sets of blood cultures were drawn and subsequently showed no growth. It is worth noting that attributing blood sterilization solely to ceftaroline therapy is difficult and that complete sterilization in MRSA bacteremia is often a long process due to the nature of staphylococcal infections. However, we had only negative blood cultures following this therapy change and noted significant clinical improvement in our patient. The patient did not have any adverse effects from therapy and continued to show clinical improvement until discharge.

\section{Discussion}

Optimal therapy for persistent MRSA bacteremia and endocarditis is now complicated by increasing concern regarding the use of daptomycin in patients failing vancomycin therapy due to observations of daptomycin MICs rising with vancomycin MICs. 'Glycopeptides and lipopeptides are the agents of choice for serious MRSA infections, but recently clinical MRSA strains have developed reduced-susceptibility phenotypes that are associated with treatment failure. ${ }^{13}$ It has been proposed that perhaps the corresponding increase in MICs between vancomycin and daptomycin is due to bacterial cell wall alterations, possibly rendering both agents ineffective against some strains of MRSA. Moreover, the recent emergence of MRSA isolates that are susceptible to vancomycin but are tolerant to its killing effects has led to a deeper analysis of the genetic variations that may exist and exert reduced susceptibility patterns. 
These vancomycin-tolerant MRSA (VT-MRSA) isolates exhibit a number of genotypic and phenotypic characteristics that may play an important role in tolerance. Additionally $S$. aureus possesses many genetic regulators, which are differentially expressed in certain strains with reduced susceptibilities to the glycoand lipopeptides. ${ }^{13}$ Gene dysfunction of the accessory gene regulator $(a g r)$ group II element reduces in vitro vancomycin killing and is associated with treatment failure in patients with severe MRSA infections. ${ }^{13}$ In daptomycin non-susceptible $S$. aureus strains, modifications in the membrane surface charge, phosphatidylglycerol turnover, and cell wall structure influence the activity of daptomycin. The expression of the $m p r F$ and/or dltABCD gene control these cell wall modifications. ${ }^{13}$ Ceftaroline targets PBP 1-4, with very high affinity for PBP2a, the protein responsible for methicillin resistance, and therefore may be able to overcome some of these resistance mechanisms. This unique affinity for this particular binding protein distinguishes ceftaroline from earlier generation cephalosporins and expands coverage to include MRSA, including the Panton-Valentine-leukocidin (PVL)-producing strains, as well as strains that show resistance to glycopeptides, daptomycin, clindamycin, linezolid, and sulfmethoxazole-trimethoprim.

Cephalosporins have been the mainstay of therapy for Gram positive infections until the recent rise in resistance to beta-lactam antibiotics, causing a shift to vancomycin as the usual first-line, empiric, agent of choice for staphylococcal infections. ${ }^{9}$ The 2009 Infectious Diseases Society of America guidelines for MRSA bacteremia recommend high-dose vancomycin with target serum troughs of $15-20 \mathrm{mcg} /$ $\mathrm{mL}$ and an AUC/MIC ratio of $>400$. $^{11}$ However, these targets have not been validated for bacteremia and achieving these targets often comes with an increased risk of nephrotoxicity. This limits its use in certain patient populations, particularly those with pre-existing renal impairment.

Ceftaroline is a novel, advanced, or "fifth generation", broad-spectrum cephalosporin that has rapid bactericidal activity against Gram-negative and
Gram-positive bacteria, including MRSA., 1,79 Ceftaroline, although not FDA approved for MRSA bacteremia or endocarditis, exhibits potent anti-MRSA activity in vitro and has low MICs for MRSA isolates. Additionally, ceftaroline has in vitro activity against vancomycin-intermediate $S$. aureus (VISA) and vancomycin-resistant strains of $S$. aureus (VRSA). ${ }^{5} \mathrm{Al}$ though human clinical trials in MRSA bacteremia or endocarditis have not been conducted, in vitro and in vivo animal models show that ceftaroline exhibits superior bactericidal activity against resistant $S$. aureus endocarditis compared to vancomycin, linezolid, and tigecycline. ${ }^{5,8,10}$ Moreover, rabbit endocarditis models have shown ceftaroline to be superior in sterilizing valvular vegetations compared to daptomycin and teicoplanin due to MRSA, MSSA (methicillin-sensitive S. aureus), and VISA. ${ }^{5,9}$ In rabbit MSSA, MRSA, and GISA (glycopeptide intermediate $S$. aureus) endocarditis models, ceftaroline was superior to daptomycin in vegetation sterilization and showed high bactericidal activity compared to tigecycline. ${ }^{10}$ The frequent monitoring that is required during vancomycin therapy, both to ensure maintenance of therapeutic trough concentrations and also to ensure lack of toxic build up, was also a challenge in treating our patient. Additionally, ceftaroline lacks the nephrotoxic effects of vancomycin or myositis associated with daptomycin, making it an attractive option for our patient and decreases the monitoring burden during therapy.

\section{Conclusion}

The increased incidence of highly resistant bacterial infections requires the use of newer drugs against those pathogens. Relying on in vitro data, successful in vivo data in animal models, and a handful of human case reports, it was decided that treatment with ceftaroline was a reasonable and safe option for our patient. Human clinical studies to establish ceftaroline's efficacy and safety profile in MRSA bacteremia and endocarditis are needed to clearly define its place in therapy, but until such studies are available we conclude that ceftaroline can be considered 
a safe alternative in persistent MRSA bacteremia and endocarditis in patients whose susceptibilities show increasing MICs to vancomycin and daptomycin or who have apparent treatment failures with first-line agents.

\section{KEYPOINTS}

1. Although the mechanism is not completely understood, there is a rising incidence of MRSA isolates that are resistant to glycopeptide and lipopeptide antimicrobial agents.

2. Ceftaroline's unique mechanism of action and target sites may explain its superior activity against MRSA isolates.

3. Although not currently FDA-approved for bacteremia or infective endocarditis, ceftaroline has robust data and case reports to support its use in these infections when treatment options are limited.

Author Affiliation: Kristen Fuhrmann is a pharmacist at University Medical Center in Lubbock TX. Paula McKenzie is a fellow in Infectious Disease in the Department of Internal Medicine at TTUHSC, Lubbock TX. Mamoun Bashir is a fellow in Nephrology at TTUHSC. Richard Winn is a faculty member in Infectious Disease at TTUHSC.

Received: 09/02/2013

Accepted: 09/25/2013

Reviewers: Vipul Desai MD, Kanokporn Mongkolrattanothai MD

Published electronically: 10/15/2013

Conflict of Interest Disclosures: None

\section{REFERENCES}

1. Ho TT, Cadena J, Childs L, et al. Methicillin-resistant Staphylococcus aureus bacteremia and endocarditis treated with ceftaroline salvage therapy. J Antimicrob Chemother 2012; 1-4.

2. Casapao A, Barber K, Wong C, et al. Early experience with ceftaroline fosamil therapy at an academic hospital system.Poster presentation ICAAC 2012.

3. Jongsma K, Joson J, Heidari A. Ceftaroline in the treatment of concominant methicillin-resistant and daptomycin-non-susceptible Staphylococcus aureus infective endocarditis and osteomyelitis: case report. $J$ Antimicrob Chemother 2013; 1-2.

4. Lin J, Aung G, Thomas A, et al. The use of cefatroline fosamil in methicillin-resistant Staphylococcus aureus endocarditis and deep-seated MRSA infections: a retrospective case series of 10 patients. J Infect Chemother 2012online publication.

5. Zainah H, Vazquez J. Use of Ceftaroline in Complicated Methicillin-Resistant Staphylococcus aureus Bacteremia. Infect Dis Clin Pract 2012; 00:00-00.

6. Walraven C, North M, Lyon-Marr L, et al. Site of infection rather than vancomycin MIC predicts vancomycin failure in methicillin-resistant Staphylococcus aureus bacteremia. J Antimicrob Chemother 2011

7. Najjar S, Biehle L, Najjar J, et al. Treatment of MethicillinResistant Staphylococcus aureus bacteremia and Prosthetic Valve Endocarditis With Ceftaroline. Infect Dis Clin Pract. 2013; 00:00-00.

8. Girish C, Balakrishnan S. Ceftaroline fosamil: A novel antiMethicillin-resistant Staphylococcus aureus cephalosporin. J Pharmacol Pharmacother 2011; 2(3):209-211

9. Saravolatz L, Stein G, Johnson L. Ceftaroline: A novel Cephalosporin with Activity against Methicillin-resistant Staphylococcus aureus. Clin Infect Dis 2011; 52115-1157.

10. Jacqueline $\mathrm{C}$, Amador $\mathrm{G}$, Batard $\mathrm{E}$, et al. Comparison of ceftaroline fosamil, daptomycin and tigecycline in an experimental rabbit endocarditis model caused by methicillin-susceptible, methicillin-resistant and glycopeptide-intermediate Staphylococcus aureus. J Antimicrob Chemother 2011; 66:863-866.

11. Liu C, Bayer A, Cosgrove SE, et al. Clinical practice guidelines by the Infectious Diseases Society of America for the treatment of methicillin-resistant Staphylococcus aureus infections in adults and children. Clin Infect Dis 2011; 52:e18-55.

12. Forest Laboratories Inc. Teflaro ${ }^{\circledR}$ Prescribing Information. St. Louis, MO, 2010-2012.

13. Rose W, Fallon M, Moran J, et al. Vancomycin Tolerance in Methicillin-Resistant Staphylococcus aureus: Influence of vancomycin, daptomycin and Telavancin on Differential Resistance Gene Expression. Antimicrob Agents Chemother 2012; 56(8)4422-4427. 